\title{
Analysis on volleyball injury improvement movement based on action screening and rehabilitation physical training.
}

\author{
Xuan Wu' ${ }^{1}$, Shangli Liu' ${ }^{2 *}$ \\ ${ }^{1}$ Key Lab of Physical Fitness Evalution and Moto Function Monitoringtion, College of Physical Education, Southwest \\ University, Chongqing, PR China \\ ${ }^{2}$ Department of Physical Education, Chongqing Institute of Science Technology, Chongqing, PR China
}

\begin{abstract}
Over the years, sport has been more and more popular. However, there's usually some athletic injury in the course of the movement, therefore, sports injuries have been more and more concerned by the community. With the development of science and technology, in all aspects, the science can be used to conduct more effective exercise. Therefore, based on researching the theoretical knowledge of action screening and rehabilitation physical training, the scientific methods were applied to improve the damage situation of volleyball, so as to make volleyball players who are injured in the movement could be able to recover more quickly.
\end{abstract}

Keywords: Action screening, Rehabilitation physical training, Volleyball injury.

Accepted on May 05, 2017

\section{Introduction}

An athlete uses hands serve, dig pass and other methods to play volleyballs, so as to attack or defend, and this sport is volleyball. It began to be popular in the late nineteenth Century in the US [1]. In the popular process, the rules of volleyball were gradually formed, and the movement was gradually welcomed by the broad masses of the people and accepted by the people of the world [2].

\section{State of the Art}

Action screening theory refers to a system for evaluating a person's physical condition and movement pattern. At the time of operation, this action screening system is very simple, and it can be easily applied to a variety of different groups of sports.

\section{Methodology}

\section{The reason for injury in volleyball}

Generally, in volleyball sport, athletes will be subject to certain damage, and this injury is usually acute injury, for example, during training, the joint injury of athletes suffered in the bounce landing. This damage is usually influenced by the growth structure of the joints of human bodies themselves and other factors.

When people are in some intense exercise, because the body can't maintain certain stability, the body is usually more accustomed to a balance, and this makes a certain bias for human body during the time of rising to the sky, under these circumstances, people will suffer the ankle injury when landing. Furthermore, the strength of the muscles around the joints and the flexibility of the ligament will affect the health of the joints, if the strength of muscles around the joints is more powerful, and the ligament of the joints has good flexibility, it can play a certain protective effect on the joints.

In addition, in the course of the athletes' exercise, because the athletes usually accept long-term exercise, it will cause some chronic strain to the athlete's joints. So that the athletes are more susceptible to injury during training or competition, and the joint stability is not strong enough, which is unable to withstand greater pressure. So the athlete's joints are more vulnerable to injury.

\section{Prevention of sports injury}

Before the athletes participate in sports training, they must learn how to protect themselves from the injury in the movement process, and improve the consciousness of selfprotection and the consciousness of preventing injury, and furthermore, athletes are asked to learn to protect themselves, so that they can fully understand the damage brought by sport injury. The chart below shows the bouncing process of volleyball (Figure 1).

In the training of volleyball players, the coach must adjust the training content, and arrange the training venue, so as to make athletes conduct the volleyball training in the most secure environment. Before the exercise, athletes should do a certain exercise preparation work, so as to prevent the athlete's joint injury. And furthermore, at the time of arranging for the 
exercise, there must be a certain degree of scientific nature and reasonable nature, so that the preparatory exercise can be more effective.

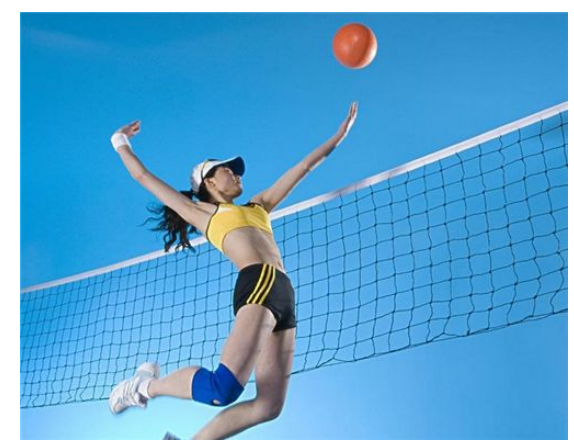

Figure 1. Process of volleyball bouncing.

After the exercise, volleyball players must carry out certain arrangement after exercise, and this can relieve the feeling of fatigue of athletes after exercise. Besides, it can also make the athletes avoid some chronic damage, and let their strength restore quickly.

\section{Result Analysis and Discussion}

As the action screening system can quickly detect the athletes' athletic indicators and make quick evaluations, this allows the athletes to effectively reduce sports injuries during exercise, and moreover, they can improve various actions of sports. Therefore, the author selects a certain number of volleyball players as research objects, collects a certain amount of data to analyze, and the specific physical data is shown in Table 1.

Table 1. Action screening system collects motion data.

\begin{tabular}{lccccc}
\hline Project & Athlete 1 & Athlete 2 & Athlete 3 & Athlete 4 & Athlete 5 \\
\hline deep squat & 76.45 & 85.46 & 87.44 & 83.45 & 84.56 \\
\hline Stepping & 73.25 & 78.36 & 73.55 & 79.24 & 69.34 \\
\hline $\begin{array}{l}\text { Straight line } \\
\text { squatting }\end{array}$ & 79.05 & 89.84 & 94.56 & 69.34 & 78.77 \\
\hline $\begin{array}{l}\text { Shoulder } \\
\text { Muscular }\end{array}$ & 89.56 & 95.01 & 87.44 & 70.56 & 93.44 \\
\hline strength & 69.34 & 93.44 & 87.34 & 89.56 & 70.45 \\
\hline $\begin{array}{l}\text { Push-up } \\
\text { Rotation }\end{array}$ & 87.44 & 70.45 & 69.34 & 81.12 & 79.98 \\
\hline
\end{tabular}

Furthermore, the data collected above is calculated and analyzed in the action screening system, and the results obtained are as follows. The main reason for the sports injury of volleyball is that the stability of each joint is insufficient, the muscle strength is not strong, and the ligaments are not soft enough, so that athletes are vulnerable to injury in the course of sports. The following figure is the data collected by the action screening system (Figure 2).

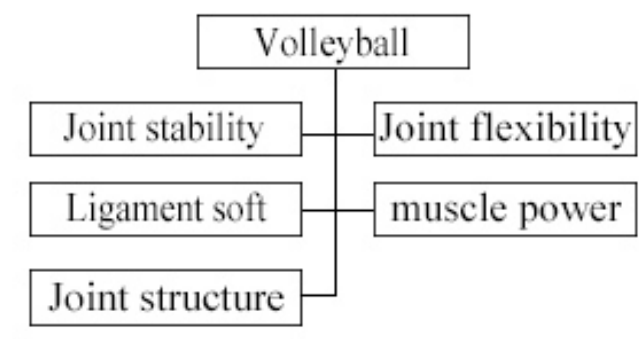

Figure 2. Various of data collected by action screening system.

The coach must conduct targeted training for athletes, strengthen the stability of athletes' joints, improve the strength of athletes' muscle, and strengthen the flexibility of athletes' ligament, thereby reducing the occurrence of injury. Moreover, in the course of exercise, it's necessary to put an end to excessive training, severe physical exertion and fatigue training and so on, so as to allow athletes to fully prepare before exercise. After the exercise, there must be a certain buffer for athletes, so that they can recover faster.

\section{Conclusions}

In the course of the movement, as the athletes often suffer from sports injuries. Therefore, in recent years, more and more studies have been carried on for sports injuries. Through various scientific theory studies, a variety of scientific means are applied to the recovery of sports injuries, which can improve the security of athletes in the training. In summary, according to study the action screening theory, the author selected a certain object of study, and summed up the method for the recovery of sports injuries, so that the countermeasures and methods for the rapid recovery of injury were put forward, which could maximize the help of athletes to improve the safety of sports, thereby providing contribution to the development of China's volleyball career.

\section{References}

1. Taube W, Lorch M, Zeiter S. Non-physical practice improves task performance in an unstable, perturbed environment: motor imagery and observational balance training. Frontiers Human Neurosci 2014; 8: 972.

2. Prigent-Tessier A, Quirié A, Maguin-Gaté K. Physical training and hypertension have opposite effects on endothelial brain-derived neurotrophic factor expression. Cardiovascular Res 2013.

\section{*Correspondence to}

Shangli Liu

Department of Physical Education

Chongqing Institute of Science Technology

PR China 